Heiko Bratke* and Børge Sivertsen

\title{
Mental and somatic health in university students with type 1 diabetes: new results from DiaSHoT18, a cross sectional national health and well-being
}

\section{survey}

https://doi.org/10.1515/jpem-2021-0041

Received January 19, 2021; accepted March 8, 2021;

published online April 12, 2021

\section{Abstract}

Objectives: To explore mental and somatic health, quality of life, alcohol-related problems, sleep problems, and diabetes related distress in university students with type 1 diabetes (T1D), compared to students without T1D. Further, we evaluated associations with gender, treatment modalities, and achieved metabolic control.

Methods: All fulltime Norwegian students aged 18-35 years pursuing higher education in 2018 ( $\mathrm{n}=162.512)$ were invited into a comprehensive national survey on health and well-being. Students that stated having diabetes was asked further questions about their diabetes care.

Results: Of 49,684 participating students, 324 participants stated having T1D. Students with T1D did not show more mental or somatic health symptoms, or report a higher level of loneliness. However, T1D was significantly associated with lower quality of life (QoL). Students with good metabolic control reached the same QoL as students without T1D. Mental disorders and suicidality were associated with lacking metabolic control. The proportion of unhealthy drinking habits was generally low, and even lower in students with T1D. Sleeping patterns were generally good, but students using continuous glucose

\footnotetext{
*Corresponding author: Heiko Bratke, Section for Pediatrics, Department of Clinical Medicine, Haugesund Hospital, Fonna Health Trust, Postbox 2170, 5504 Haugesund, Norway; Section for Pediatrics, Department of Clinical Medicine, University of Bergen, Bergen, Norway; and Department of Research and Innovation, Fonna Health Trust, Haugesund, Norway, Phone: +47 92051592,

E-mail: heiko.bratke@uib.no. https://orcid.org/0000-0001-5062 3271

Børge Sivertsen, Department of Health Promotion, Norwegian Institute of Public Health, Bergen, Norway; Department of Research and Innovation, Fonna Health Trust, Haugesund, Norway; and Department of Mental Health, Faculty of Medicine and Health Sciences, Norwegian University of Science and Technology, Trondheim, Norway. https://orcid.org/0000-0003-4654-9296
}

measurement were awakening more often during sleep. Females with T1D showed higher levels of diabetes related problems and distress, but good metabolic control was associated with lower diabetes distress level.

Conclusions: Students with T1D scored equally on most mental and somatic health scales. Their quality of life was significantly worse compared to their healthy peers. Knowledge on the impact of metabolic control, gender and the use of CSII and CGM can be valuable for the caretakers of young adults with T1D.

Keywords: alcohol; CGM; CSII; diabetes mellitus; mental health; quality of life; sleep; students; type 1; young adult.

\section{Introduction}

Life with type 1 diabetes (T1D) is demanding in many ways. The attempt to keep blood glucose in an acceptable range, to avoid acute and chronic complications, can be both time consuming and cognitively challenging. Norway has one of the highest incidence rates of T1D in the world [1]. Consequently, the proportion of university students with T1D in Norway is relatively high. The incidence of T1D has been increasing over the last decades, and is expected to increase further in the future [2].

Norwegian university students experience quite optimal conditions for their studies, and support in the management of their disease: Young adults in Norway have access to education free of tuition fees at public universities. The Norwegian State Educational Loan Fund provides loans and grants to all university students to give the same possibilities for education, regardless of economic and social background, age, gender, and physical disabilities. The Norwegian health care system covers all eventual costs for medical consultations and treatment, including continuous glucose monitoring (CGM) and insulin pumps, exceeding approximately $\$ 200$ per year.

Nevertheless, there is reason to believe that T1D can lead to secondary mental and somatic health problems. A higher degree of anxiety and poorer quality of life (QoL) 
have been reported, and an increase in depressive symptoms from adolescence to early adulthood in students [3-5].

The primary aim of this study was to compare the occurrence of symptoms related to mental and somatic health, mental disorders, QoL, loneliness, positive affect, alcohol-related problems, and sleep problems between university students with T1D vs. those without T1D. Secondary aims were to identify possible associations to gender, the use of diabetes treatment modalities, and achieved metabolic control.

\section{Materials and methods}

In 2018, a cross sectional national survey of all students pursuing higher education in Norway was conducted (the SHoT2018 study (Students' Health and Wellbeing Study) [6]. The overall aim of the SHoT study was to examine the prevalence and trends across a range of health problems and life challenges among college and university students. Since T1D has a high prevalence in Norway, it was also part of the survey to illuminate the specific challenges and concerns of students with this disease.

\section{Procedure}

The SHoT2018 survey was conducted between February 6 and April 5, 2018. All fulltime Norwegian students aged 18-35 years pursuing higher education were invited to participate. In all, 162,512 students fulfilled these inclusion criteria, of which 49,684 students completed the relevant questionnaires used in the present study, yielding a response rate of $31 \%$. Detailed information on the procedure has already been published [6, 7].

\section{Instruments}

\section{Somatic health symptoms}

Somatic health was assessed by the Somatic Symptom Scale-8 (SSS-8): An 8-item reliable and valid self-report measure of somatic symptom burden originally derived from the well-validated PHQ-15 [8]. The Cronbach's alpha for the SSS-8 in the current study was 0.82 .

\section{Mental health symptoms}

Mental health symptoms were assessed using The Hopkins Symptoms Checklist (HSCL-25) [9], a screening tool designed to detect symptoms of anxiety and depression. It is composed of a 10-item subscale for anxiety and a 15-item subscale for depression, with each item scored on a Likert scale from 1 ("not at all") to 4 ("extremely"). Previous studies have shown that an unidimensional model had the best psychometric properties in the student population and not the original subscales of anxiety and depression [10]. Consequently, we chose to compute a single total scale representing common mental health symptoms in the present study. The Cronbach's alpha for the HSCL-25 in the current study was 0.94 .

\section{Mental disorders}

Self-reported mental disorders were assessed using the same predefined list that was used to identify diabetes. The list was based on a similar operationalization used in previous large population-based studies (the HUNT study [11]), and included several subcategories for most conditions/disorders. In the present study, only anxiety and depressive disorder were used. The rationale for including both the HSCL-25 and the items of self reported anxiety and depression, was to provide both an assessment of overall symptom load (from the HSCL-25), and an indication of the presence of depression and anxiety.

\section{Suicidal ideation, suicidal behavior, and self harm}

History of suicidal ideation, suicide attempts, and nonsuicidal self harm (NSSH) were assessed with 3 items drawn from the Adult Psychiatric Morbidity Survey (APMS) [12]; "Have you ever seriously thought of taking your life, but not actually attempted to do so?", "Have you ever made an attempt to take your life, by taking an overdose of tablets or in some other way?", and "Have you ever deliberately harmed yourself in any way but not with the intention of killing yourself? (i.e., self-harm)," respectively. The questions about self harm thoughts were adapted from the Child and Adolescent Self harm in Europe (CASE) study [13]. "Have you ever seriously thought about trying to deliberately harm yourself but not with the intention of killing yourself but not actually done so?” (Yes/no). The Cronbach's alpha for the 4 items was 0.72 .

\section{Loneliness}

Loneliness was assessed using an abbreviated version of the widely used UCLA Loneliness Scale, "The ThreeItem Loneliness Scale (T-ILS)" [14]. The T-ILS include 
the following 3 items, each rated along a 5-point Likert scale ( $1=$ "never", 5 = "very often"). For each question below, please indicate how often you have felt that way during the last year: 1) How often do you feel that you lack companionship? 2) How often do you feel left out? and 3) How often do you feel isolated from others? Higher scores indicate higher level of experienced loneliness. The Cronbach's alpha for the T-ILS in the present study was 0.88 .

\section{Positive affect}

The Positive and Negative Affect Schedule (PANAS) [15] is a 20-item questionnaire with two subscales, measuring positive and negative affect (PA/NA). The NA subscale was not included in this study. Participants were instructed to rate to what extent they experience each emotion right now, rated on a 5-point scale from ( 1 = "very slightly or not at all" to $5=$ "extremely"). A sum score was calculated with higher scores representing greater positive affect. The Cronbach's alpha for the positive affect subscale in the present study was 0.91 .

\section{Quality of life}

Quality of life was assessed by the Satisfaction with Life Scale (SWLS) [16]. The SWLS is a 5-item scale designed to measure global cognitive judgments of one's life satisfaction (not a measure of either positive or negative affect). Participants indicate how much they agree or disagree with each of the 5 items using a 7-point scale from 7 (strongly agree) to 1 (strongly disagree). Higher scores indicate higher QoL. Cronbach's alpha for the SWLS in the present study was 0.89 .

\section{Alcohol-related problems}

Potential alcohol-related problems were measured by the Alcohol Use Disorders Identification Test (AUDIT), which is a widely used instrument developed by the World Health Organization for identifying risky or harmful alcohol use $[17,18]$. The 10-item AUDIT includes items for measuring the frequency, typical amount and episodic heavy drinking frequency (items 1-3), alcohol dependence (items 4-6) and problems related to alcohol consumption (items 7-10) [19]. A score of eight or more is associated with harmful or hazardous drinking. Cronbach's alpha for the SWLS in the present study was 0.76 .

\section{Sleep variables}

The participants' self-reported usual bedtime and rise time were indicated in hours and minutes, and data were reported separately for weekdays and weekends. Time in bed (TIB) was calculated as the difference between bedtime and rise time. Sleep onset latency (SOL) and wake after sleep onset (WASO) were indicated in hours and minutes. Sleep duration was defined as TIB minus SOL and WASO.

All participants also indicated the number of nights per week they experienced difficulties initiating sleep (DIS), difficulties maintaining sleep (DMS), and early morning awakenings (EMA), and daytime sleepiness and tiredness. Those suffering from sleep problems were asked how long the problems had been present. The following three criteria were used as an operationalization for insomnia disorder [20], in line with the DSM-5 criteria: 1) the presence of either DIS, DMS, or EMA for at least three nights per week, 2) the presence of daytime sleepiness and tiredness for at least three days per week, and 3) a duration of the sleep problems for at least three months.

\section{Diabetes-related characteristics}

Physical conditions were assessed by a predefined list adapted to fit this age cohort, which also included "diabetes". If the student indicated "yes" for diabetes, several additional questions would be asked, as described previously [7]. In short, students stated their type of diabetes and their last HbA1c value. They also reported whether they used an insulin pen or pump, and whether they used CGM or not. Students were also asked about the frequency of their blood glucose measurements.

In addition, all students reporting to have diabetes completed two brief questionnaires assessing diabetesrelated distress: the problem areas in diabetes scale (PAID-1) [21] and the diabetes distress scale (DDS2) [22].

At the time of data collection, an HbA1c under $58 \mathrm{mmol} / \mathrm{mol}(7.5 \%)$ was the recommended treatment goal from the International Society of Pediatric and Adolescent Diabetes (ISPAD). We therefore categorized our study sample into students reporting an HbA1c value under $58 \mathrm{mmol} / \mathrm{mol}(7.5 \%)$ and students with an HbA1c of $58 \mathrm{mmol} / \mathrm{mol}(7.5 \%)$ or higher.

\section{Statistical analysis}

We used IBM SPSS Statistics 26 for Windows (SPSS Inc., Chicago, IL) for all analyses. Chi-squared tests and 
independent samples t-tests were used to examine differences on the outcome measures among students with and without T1D. We also compared T1D students achieving the HbA1c treatment goal ( $58 \mathrm{mmol} / \mathrm{mol}, 7.5 \%$ ) to students not achieving the treatment goal. Sum scores were converted to standardized t-scores to ease the comparison across the instruments, and between-group effect sizes (pooled SD) were calculated using the Cohen's d formula [23]. Estimated marginal means (adjusting for age of diabetes onset) were computed for diabetes-related problems and distress in males and females with T1D by HbA1c treatment goal. We also examined diabetes-related problems and distress by demographic and diabetes-related variables. There was generally little missing data, and hence missing values were handled using listwise deletion.

\section{Ethics}

The SHoT2018 study was approved by the Regional Committee for Medical and Health Research Ethics in Norway (no. 2017/1176). An electronic informed consent was obtained after the participants had received a detailed introduction to the study.

\section{Results}

\section{Mental and somatic health}

As displayed in Figure 1, university students with T1D did on a general level not differ from students without T1D on most of the health domains, with similar levels of mental health symptoms, somatic symptoms, loneliness, and positive affect. However, students with T1D reported lower quality of life (Cohen's $d=0.18, p<0.01$ ), and fewer alcohol problems (Cohen's $d=0.17, p<0.01$ ) than students without T1D. Significantly fewer students with T1D had an alcohol use classified as problematic or harmful (AUDIT >8) compared to students without T1D (47.2 vs. 56.5\%, $\mathrm{p}<0.001)$.

Compared to students not achieving the HbA1c treatment goal ( $58 \mathrm{mmol} / \mathrm{mol}, 7.5 \%)$, students achieving this goal scored better across all health questionnaires. Achieving the treatment goal was associated with fewer mental health symptoms, less somatic symptoms, less loneliness, and higher levels of quality of life and positive affect (Figure 1).

Importantly, compared with students without T1D, students with T1D who did achieve the HbA1c treatment goal had significantly fewer mental health symptoms $(\mathrm{p}=0.008)$ and somatic symptoms ( $\mathrm{p}=0.009)$, and also scored significantly better on positive affect measure $(p=0.012)$. The groups did not differ significantly on reported quality of life and loneliness.

\section{Mental disorders and suicidality}

There was no significant difference between students with T1D compared to those without T1D regarding mental disorders and suicidality (Table 1). However, students not achieving the HbA1c treatment goal had higher rates of both anxiety and depressive disorders, and NSSH, NSSH thoughts and suicidal thoughts, compared to students achieving the HbA1c treatment goal (Table 1).

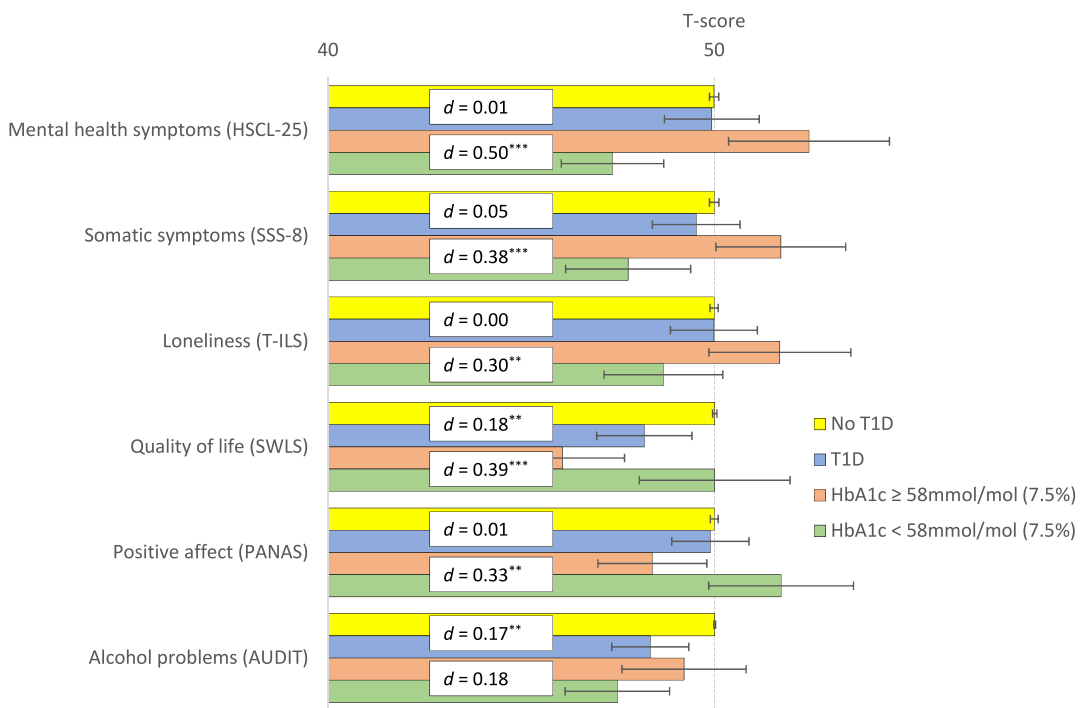

Figure 1: Somatic and mental health among students with and without T1D, and by HbA1c treatment goal (Estimates represented in t-scores (in bars) and Cohen's d effect size (in white text box). Error bars represent $95 \%$ confidence intervals. ${ }^{* \star *} p<0.001 ;{ }^{* \star} p<0.01 ;{ }^{*} p<0.05$. 
Table 1: Mental disorders, insomnia, and suicidality among students with and without T1D, and by HbA1c treatment goal ( $58 \mathrm{mmol} / \mathrm{mol}$, $7.5 \%)$.

\begin{tabular}{|c|c|c|c|c|c|c|c|c|c|c|}
\hline & \multicolumn{2}{|c|}{ No T1D $(n=49,684)$} & \multicolumn{2}{|c|}{ T1D $(n=324)$} & \multirow[t]{2}{*}{ p-Value } & \multicolumn{2}{|c|}{$\begin{array}{r}\mathrm{HbA1c}<58 \mathrm{mmol} / \mathrm{mol} \\
(7.5 \%)(\mathrm{n}=185)\end{array}$} & \multicolumn{2}{|c|}{$\begin{array}{r}\text { HbA1c } \geq 58 \mathrm{mmol} / \mathrm{mol} \\
(7.5 \%)(\mathrm{n}=167)\end{array}$} & \multirow[t]{2}{*}{ p-Value } \\
\hline & $\%$ & (n) & $\%$ & (n) & & $\%$ & (n) & $\%$ & (n) & \\
\hline Anxiety disorder & $10.1 \%$ & $(5,012)$ & $9.6 \%$ & (31) & NS & $7.6 \%$ & (14) & $14.4 \%$ & (24) & 0.040 \\
\hline Depressive disorder & $11.1 \%$ & $(5,510)$ & $12.0 \%$ & (39) & NS & $7.6 \%$ & (14) & $19.2 \%$ & (32) & 0.001 \\
\hline Insomnia disorder & $30.5 \%$ & $(11,151)$ & $32.1 \%$ & (104) & NS & $29.7 \%$ & (55) & $38.3 \%$ & (64) & NS \\
\hline NSSH & $19.7 \%$ & $(9,746)$ & $19.2 \%$ & $(62)$ & NS & $15.8 \%$ & (29) & $25.1 \%$ & (42) & 0.029 \\
\hline NSSH thoughts & $22.7 \%$ & $(112,076)$ & $22.8 \%$ & (74) & NS & $17.3 \%$ & (32) & $34.1 \%$ & (57) & $<0.001$ \\
\hline Suicide attempt & $4.2 \%$ & (2093) & $4.3 \%$ & (14) & NS & $4.9 \%$ & (9) & $6.6 \%$ & (11) & NS \\
\hline Suicide thought & $21.0 \%$ & $(10,404)$ & $23.2 \%$ & (75) & NS & $20.0 \%$ & (37) & $29.5 \%$ & (49) & 0.038 \\
\hline
\end{tabular}

\section{Sleep problems}

As detailed in Table 2, there were no significant differences between students with and without T1D across most sleep parameters, with the exception of 10-13 min later reported bedtimes in the group with T1D. Achieving the HbA1c treatment goal was associated with earlier bedtimes and rise times (week days and weekends), shorter sleep onset latency, and higher sleep efficiency. No group differences were observed for time in bed, sleep duration or time wake after sleep onset. Insulin pump usage was not associated with any of the sleep measures. However, students using CGM had significantly longer time awakening after sleep onset (WASO) than non users, both on weekdays ( 25 vs. $13 \mathrm{~min}, \mathrm{p}<0.05)$ and weekends (22 vs. $8 \mathrm{~min}, \mathrm{p}<0.05)$. CGM users also had significantly longer sleep onset latency (SOL) on weekends (45 vs. $34 \mathrm{~min}$, $\mathrm{p}<0.05)$, and shorter weekend sleep duration (8:13 vs. $8: 37 \mathrm{~h} \mathrm{p}<0.05)$.

\section{Diabetes-related problems and distress}

In terms of worrying about the future and the possibility of serious complications (PAID-1), female students with T1D had the overall highest level of worry, especially women not achieving the HbA1c treatment goal (Figure 2). The same pattern was observed for the DDS-2 item assessing feeling of failure with the diabetes routine. Males achieving the treatment goal had the least amount of distress, while women not meeting the treatment goal had the most. Regarding feeling overwhelmed with the demands of living with diabetes, a similar trend was observed, with the exception of males - where reaching the HbA1c treatment goal did not affect the level of distress.
The overall level of diabetes-related distress and problems across demographic and other diabetes-related variables are displayed in Figure 3. High level of distress was associated with being female, and not achieving the HbA1c treatment goal. The level of distress was inversely associated with blood glucose measurements, the less frequent the measurement, the higher the distress. Usage of CGM or insulin pumps was not associated with the level of distress.

\section{Discussion}

In this national student survey for higher education in Norway, we examined different aspects of the mental and somatic health of university students with T1D. We compared not only students with T1D with healthy controls, but also evaluated associations with gender, metabolic control (HbA1c), and treatment devices (insulin pumps and CGM) in the group of students with T1D. Existing knowledge on the experienced somatic and mental health problems among students with T1D is limited, and based on qualitative studies or studies with few participants $[3,5]$. With our study, we supply detailed data from a large cohort of students with T1D.

The study has several strengths: We provide a large reference group of approximately 50.000 students. The survey includes a wide range of detailed quantitative data regarding somatic and mental health, including gender aspects, level of glycemic control, and use of technical devices. The percentage of students with T1D in our cohort $(0.65 \%)$ is in line with the prevalence of the disease in the Norwegian population [24]. Male and female participation ( 0.8 vs. $0.6 \%$ ) reflects the known difference in the incidence of T1D regarding gender [1]. Also, the large proportion of female students in our cohort is in line with the 
Table 2: Sleep characteristics among students with and without T1D, and by HbA1c treatment goal ( $58 \mathrm{mmol} / \mathrm{mol}, 7.5 \%$ ).

\begin{tabular}{|c|c|c|c|c|c|c|c|c|c|c|}
\hline & \multicolumn{2}{|c|}{ No T1D $(n=49,684)$} & \multicolumn{2}{|c|}{ T1D $(n=324)$} & \multirow[t]{2}{*}{ p-Value } & \multicolumn{2}{|c|}{$\begin{array}{r}\text { HbA1c }<58 \mathrm{mmol} / \mathrm{mol} \\
(7.5 \%)(\mathrm{n}=185)\end{array}$} & \multicolumn{2}{|c|}{$\begin{array}{l}\text { HbA1c } \geq 58 \mathrm{mmol} / \mathrm{mol} \\
(7.5 \%)(n=167)\end{array}$} & \multirow[t]{2}{*}{ p-Value } \\
\hline & $\begin{array}{r}\text { Mean } \\
\text { hr:min }\end{array}$ & $\begin{array}{r}\text { SD } \\
\text { hr:min }\end{array}$ & $\begin{array}{r}\text { Mean } \\
\text { hr:min }\end{array}$ & $\begin{array}{r}\text { SD } \\
\text { hr:min }\end{array}$ & & $\begin{array}{r}\text { Mean } \\
\text { hr:min }\end{array}$ & $\begin{array}{r}\text { SD } \\
\text { hr:min }\end{array}$ & $\begin{array}{r}\text { Mean } \\
\text { hr:min }\end{array}$ & $\begin{array}{r}\text { SD } \\
\text { hr:min }\end{array}$ & \\
\hline \multicolumn{11}{|l|}{ Weekdays } \\
\hline Bedtime & $23: 15$ & 01:01 & $23: 28$ & 01:10 & $<0.001$ & $23: 13$ & $0: 54$ & $23: 40$ & $1: 23$ & $<0.001$ \\
\hline Risetime & $07: 43$ & 01:17 & $07: 51$ & 01:29 & NS & 7:39 & $1: 14$ & $8: 03$ & $1: 42$ & 0.013 \\
\hline Time in bed & $08: 28$ & 01:07 & $08: 23$ & $01: 15$ & NS & $8: 26$ & $1: 00$ & $8: 22$ & $1: 29$ & NS \\
\hline Sleep onset latency & $00: 48$ & $00: 43$ & $00: 51$ & $00: 48$ & NS & $0: 45$ & $0: 45$ & $0: 56$ & $0: 49$ & 0.017 \\
\hline $\begin{array}{l}\text { Wake after sleep } \\
\text { onset }\end{array}$ & $00: 15$ & $00: 34$ & $00: 16$ & $00: 32$ & NS & $0: 11$ & $0: 19$ & $0: 22$ & $0: 46$ & 0.063 \\
\hline Sleep duration & $07: 24$ & $01: 25$ & $07: 15$ & $01: 36$ & NS & $7: 29$ & $1: 18$ & 7:02 & $1: 51$ & NS \\
\hline $\begin{array}{l}\text { Sleep efficiency, \% } \\
\text { Weekends }\end{array}$ & $87.3 \%$ & 14.3 & $86.2 \%$ & 14.6 & NS & 88.6 & 11.2 & 83.6 & 17.2 & 0.014 \\
\hline Bedtime & $00: 40$ & 01:18 & $00: 50$ & 01:18 & 0.042 & $00: 35$ & $1: 11$ & 01:00 & $1: 27$ & 0.003 \\
\hline Risetime & 09:52 & $01: 24$ & $10: 10$ & 01:31 & 0.001 & $9: 56$ & $1: 24$ & $10: 21$ & $1: 36$ & 0.004 \\
\hline Time in bed & 09:12 & 01:11 & 09:19 & 01:11 & NS & $9: 20$ & $0: 59$ & $9: 21$ & $1: 21$ & NS \\
\hline Sleep onset latency & $00: 34$ & $00: 37$ & $00: 37$ & $00: 42$ & NS & $0: 33$ & $0: 39$ & $0: 42$ & $0: 44$ & 0.018 \\
\hline $\begin{array}{l}\text { Wake after sleep } \\
\text { onset }\end{array}$ & $00: 12$ & $00: 33$ & $00: 12$ & $00: 27$ & NS & $0: 09$ & $0: 18$ & $0: 17$ & $0: 42$ & NS \\
\hline Sleep duration & $08: 24$ & $01: 26$ & $08: 30$ & $01: 27$ & NS & $8: 38$ & 1:09 & $8: 21$ & $1: 39$ & NS \\
\hline Sleep efficiency, \% & $91.3 \%$ & 11.8 & $91.0 \%$ & 10.5 & NS & 92.52 & 8.09 & 89.35 & 12.49 & 0.058 \\
\hline
\end{tabular}

Tests are adjusted for all pairwise comparisons using the Benjamini-Hochberg correction.

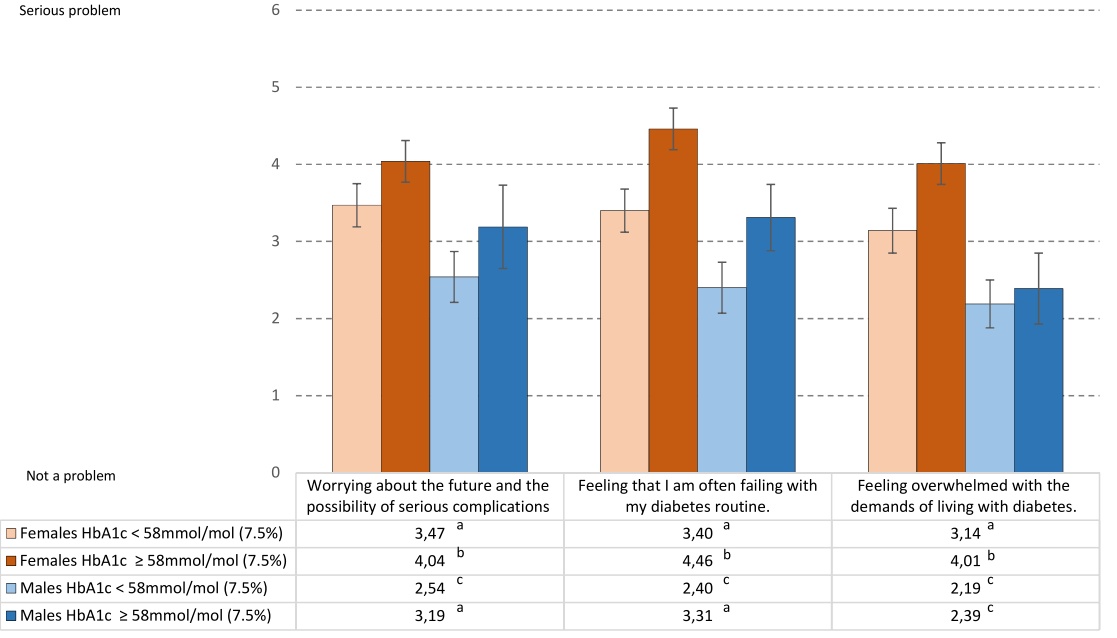

Figure 2: Problems (PAID-1) and distress (DDS-2) in males and females with type 1 diabetes (T1D) by HbA1c. Error bars represent $95 \%$ confidence intervals. Note: The superscript letters ( $a, b$, and $c)$ behind the values are used to indicate pairwise comparisons and whether these comparisons are statistically different. Values in the same row not sharing the same subscript letter are significantly different at $p<0.05$ (adjusted for multiple comparisons using the Benjamini-Hochberg correction).
Norwegian student community. Our data should therefore be representative for the Norwegian student group. As a limitation of the study, we have a modest response rate of $31 \%$. Further, our results might give an underestimation of symptoms, since non participants of health surveys tend to have worse health than participants [25].

\section{Mental and somatic health}

Chronic diseases such as T1D are likely to affect quality of life. Students with T1D reported a lower quality of life compared to their healthy peers. A previous study in adolescents aged 13-19 years with type 1 diabetes showed no differences in QoL compared to healthy peers [26]. During transition to adulthood, the perceived QoL might change because of the diverse challenges of being a young adult and additionally a university student. However, students with well-regulated T1D reported a quality of life equal to those without diabetes. It is reassuring that students with T1D did not have more mental health problems or somatic symptoms, or report a higher level of loneliness. Students with good metabolic control had even better scores than their healthy 


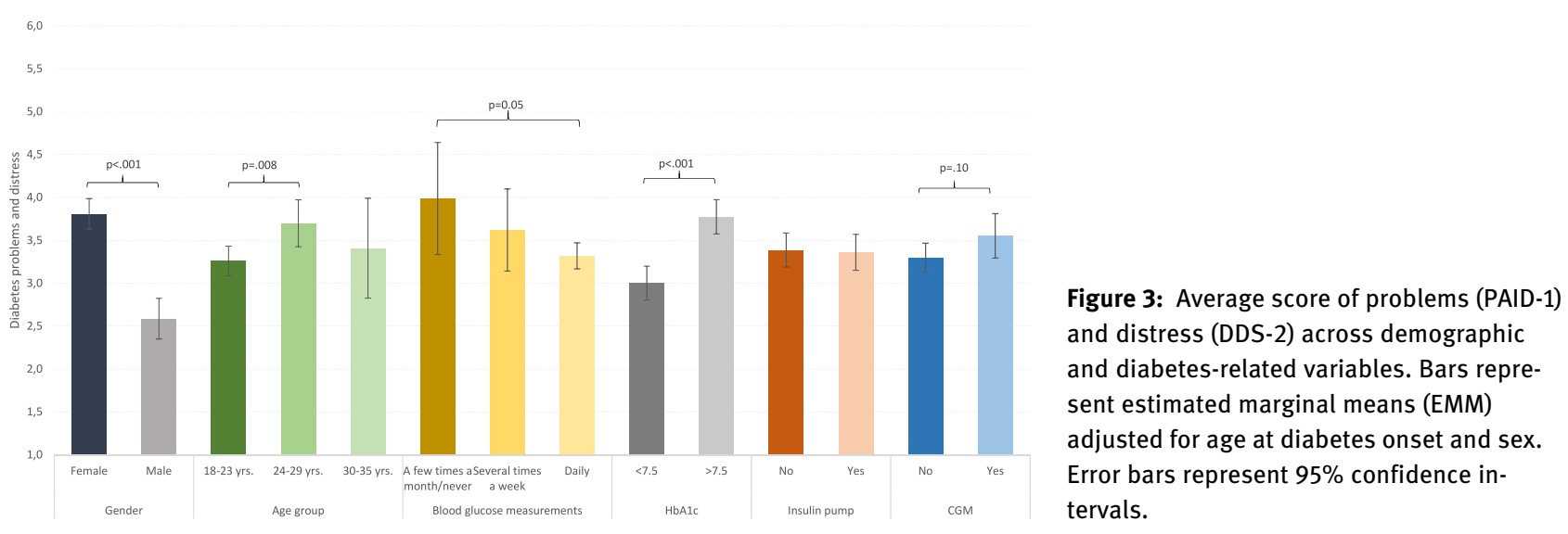

peers in some areas, such as mental health problems, somatic symptoms, and loneliness.

In contrary to existing data from other countries, problematic alcohol use was less common in Norwegian students with T1D [27, 28]. A review by Pastor et al. (2017) has shown partly dangerous and excessive drinking habits in subjects with T1D, mostly in a higher degree than in the general population, but at least at the same level [27]. Alcohol misuse is a major factor for premature death, known to account for approximately $10 \%$ of deaths among adults in the United States [29]. Lunstead et al. (2019) reported that even though a majority of adolescents with T1D were asked about their alcohol use by a healthcare provider, only $2 \%$ reported receiving the message "I should not drink" [30]. The low incidence of alcohol related problems in our total cohort, but even lower incidence in the group of students with diabetes, might imply that the Norwegian attempts to address this problematic area during follow-up in adolescent diabetes care are effective. This is of special interest, given the paucity of evidence on interventions to minimize the risks of alcohol in young adults with diabetes [31].

\section{Mental disorders and suicidality}

In line with published meta analyses [32, 33], our data did not show an increased occurrence of anxiety or depression in students with T1D on a general level. However, our data supply to existing knowledge by showing that anxiety, depressive disorders, nonsuicidal self harming thoughts, and suicidal thoughts were significantly more common in students who could not reach the HbA1c target.

\section{Sleep problems}

Sleep is important to master challenges during the day. The demanding self management of T1D, but also acute complications such as hypoglycemia or psychological aspects can lead to disturbed sleep patterns (restriction, deprivation, and fragmentation) [34]. Sleep quality has been reported to be poorer in both children and adults with T1D [35, 36]. In our study, we could observe that students both with and without T1D obtained the recommended sleeping duration of 7-9 $\mathrm{h}$ [37]. Our data are in line with a review from Griggs et al. (2019), describing sleep duration in young adults with T1D to be comparable to healthy controls. A connection between poor sleep quality in adolescents with T1D and increased HbA1c has also been shown recently [38]. Our data are in line with the results from von Schnurbein et al. (2018) showing a connection between reaching an $\mathrm{HbA1c}$ below 7.5\% (58 mmol $/ \mathrm{mol}$ ) and shorter sleep onset latency, and higher sleep efficiency.

Knowledge on the use of CGM and sleeping patterns is still limited. Jaser et al. (2017) reported no correlation between the use of CGM or insulin pumps and the sleep of children with T1D [39]. A newly published brief report by Sinisterra et al. (2020) focused on the use of CGM and sleep in young children with T1D [40]. Their data on CGM were more nuanced, showing that the child's sleep was less disturbed under the use of CGM, whereas the parents' sleep was more disturbed. Our results on university students' use of CGM are in line with the later: CGM users needed longer to start sleeping, were more awake during sleep, and in all slept shorter. If the observed differences in sleep were caused by important information by the CGM system, and resulted in a reaction by the student that improved the quality of diabetes selfmanagement, the benefit of CGM would outweigh the resulting differences in sleep. We believe that a large amount of sleep disturbances by CGM can be valuable, and sleeping through e.g., a hypoglycemic episode would be the worse alternative. As an implication for the clinician, the CGM related differences in sleeping patterns should be addressed with the patient, 
and possible unnecessary nightly alarms and disturbance by the device should be critically evaluated. Exact data on the reason for the described differences in sleep in the CGM user group are lacking, and could be the focus of future studies.

\section{Diabetes-related problems and distress}

We observed noticeable results on the level of diabetesrelated problems and distress. Both worrying about the future and the possibility of complications and the feeling of failing with diabetes routines were associated with reaching or not-reaching the HbA1c treatment goal. The use of CSII or CGM was not associated with less diabetes related problems or distress. However, there was a clear gender difference. Females reported, even with a well regulated $\mathrm{HbA1c}$, more diabetes related problems and distress compared to their male peers (Figure 2). This is important for the clinician taking care of the young adults with T1D. Worries and diabetes related distress have to be addressed differently when mediating the results of self management to their patients, knowing about these differences. The female group might need more reassurance when actually coping very well in managing their diabetes, whereas males might underestimate the consequences of failing with diabetes routines.

As this national student survey for higher education in Norway shows, university students with T1D can live their lives equally compared to their healthy peers, regarding most aspects of mental and somatic health. However, students with T1D reported lower QoL compared to students without T1D. Lower HbA1c was associated with higher scores across most health questionnaires, including fewer mental and somatic health problems, less loneliness, higher QoL, and a more positive affect. Good metabolic control as reflected by low HbA1c is therefore a critical parameter for good mental and somatic health among students with T1D.

Acknowledgements: We thank the students for participating in the SHoT2018 survey, as well as the three largest student welfare organizations in Norway (SiO, Sammen, and SiT), who initiated and designed the SHoT study.

Research funding: None declared.

Author contributions: B.S. is the guarantor of this work and, as such, had full access to all the data in the study and takes responsibility for the integrity of the data and the accuracy of the data analysis. H.B. and B.S. took the initiative for the study. B.S. is responsible for the collection of the data in SHoT2018. H.B. and B.S. contributed to the analysis and interpretation of the data, and the manuscript development, vouch for the accuracy and completeness of the data reported, and made the decision to submit the manuscript for publication. H.B. and B.S. finally approve this version to be published. All authors have accepted responsibility for the entire content of this manuscript and approved its submission.

Competing interests: Authors state no conflict of interest. Informed consent: An electronic informed consent was obtained after the participants had received a detailed introduction to the study.

Ethical approval: The SHoT2018 study was approved by the Regional Committee for Medical and Health Research Ethics in Norway (no. 2017/1176).

\section{References}

1. Skrivarhaug T, Kummernes SJ, Kamaleri Y. The Norwegian Childhood Diabetes Registry (NCDR) annual report 2018; 2019. Available from: https://oslo-universitetssykehus.no/seksjonavdeling/Documents/2019.10.01_Endelig_Årsrapport_2018. pdf.

2. Xia Y, Xie Z, Huang G, Zhou Z. Incidence and trend of type 1 diabetes and the underlying environmental determinants. Diabetes Metab Res Rev 2019;35:e3075.

3. Ersig AL. An exploratory study: transition to adulthood for college students with type 1 diabetes and their parents. J Pediatr Nurs 2019;46:12-7.

4. Wisk LE, Weitzman ER. 57. Psychosocial distress and resilience among college students with type 1 diabetes. J Adolesc Health 2019;64:S31-2.

5. Fredette J, Mawn B, Hood K, Fain J. Quality of life of college students living with type 1 diabetes: a qualitative view. West J Nurs Res 2016;38:1595-610.

6. Sivertsen B, Råkil H, Munkvik E, Lønning KJ. Cohort profile: the SHoT-study, a national health and well-being survey of Norwegian university students. BMJ Open 2019;9.

7. Bratke H, Lonning KJ, Sivertsen B. DiaSHoT18: a cross-sectional national health and well-being survey of university students with type 1 diabetes. Pediatr Diabetes 2020;21.

8. Spitzer RL, Kroenke K, Williams JB. Validation and utility of a selfreport version of PRIME-MD: the PHQ primary care study. Primary care evaluation of mental disorders. Patient health questionnaire. JAMA 1999;282:1737-44.

9. Derogatis LR, Lipman RS, Rickels K, Uhlenhuth EH, Covi L. The Hopkins Symptom Checklist (HSCL): a self-report symptom inventory. Behav Sci 1974;19:1-15.

10. Skogen JC, Øverland S, Smith OR, Aarø LE. The factor structure of the Hopkins Symptoms Checklist (HSCL-25) in a student population: a cautionary tale. Scand J Publ Health 2017;45: 357-65.

11. Krokstad S, Langhammer A, Hveem K, Holmen TL, Midthjell K, Stene TR, et al. Cohort profile: the HUNT study, Norway. Int J Epidemiol 2013;42:968-77. 
12. McManus S, Bebbington P, Jenkins R, Brugha T. Mental health and wellbeing in England: adult psychiatric morbidity survey 2014. Leeds: NHS Digital; 2016.

13. Madge N, Hewitt A, Hawton K, de Wilde EJ, Corcoran P, Fekete S, et al. Deliberate self-harm within an international community sample of young people: comparative findings from the Child \& Adolescent Self-harm in Europe (CASE) Study. J Child Psychol Psychiatry 2008;49:667-77.

14. Hughes ME, Waite LJ, Hawkley LC, Cacioppo JT. A short scale for measuring loneliness in large surveys: results from two population-based studies. Res Aging 2004;26:655-72.

15. Watson D, Clark LA, Tellegen A. Development and validation of brief measures of positive and negative affect: the PANAS scales. J Pers Soc Psychol 1988;54:1063-70.

16. Diener E, Emmons RA, Larsen RJ, Griffin S. The satisfaction with life scale. J Pers Assess 1985;49:71-5.

17. Babor TF, Higgins-Biddle JC, Saunders JB, Monteiro MG. The alcohol use disorders identification test (AUDIT) manual: guidelines for use in primary care. In: Dependence DoMHaS, editor. WHO/MSD/MSB/01.6a. Geneva: World Health Organization; 2001.

18. Saunders JB, Aasland OG, Babor TF, de la Fuente JR, Grant M. Development of the alcohol use disorders identification test (AUDIT): WHO collaborative project on early detection of persons with harmful alcohol consumption-II. Addiction 1993;88: 791-804.

19. Shevlin M, Smith GW. The factor structure and concurrent validity of the alcohol use disorder identification test based on a nationally representative UK sample. Alcohol 2007;42:582-7.

20. Sivertsen B, Vedaa Ø, Harvey AG, Glozier N, Pallesen S, Aarø LE, et al. Sleep patterns and insomnia in young adults: a national survey of Norwegian university students. J Sleep Res 2019;28: e12790.

21. McGuire BE, Morrison TG, Hermanns N, Skovlund S, Eldrup E, Gagliardino J, et al. Short-form measures of diabetes-related emotional distress: the problem areas in diabetes scale (PAID)-5 and PAID-1. Diabetologia 2010;53:66-9.

22. Fisher L, Glasgow RE, Mullan JT, Skaff MM, Polonsky WH. Development of a brief diabetes distress screening instrument. Ann Fam Med 2008;6:246-52.

23. Cohen J. Statistical power analysis for the behavioral sciences, 2nd ed. Mahwah, New Jersey: Lawrence Erlbaum Associates; 1988.

24. Strom H, Selmer R, Birkeland KI, Schirmer H, Berg TJ, Jenum AK, et al. No increase in new users of blood glucose-lowering drugs in Norway 2006-2011: a nationwide prescription database study. BMC Publ Health 2014;14:520.

25. Knudsen AK, Hotopf M, Skogen JC, Overland S, Mykletun A. The health status of nonparticipants in a population-based health study: the Hordaland Health Study. Am J Epidemiol 2010;172: 1306-14.
26. Lukacs A, Sasvari P, Torok A, Barkai L. Generic and diseasespecific quality of life in adolescents with type 1 diabetes: comparison to age-matched healthy peers. J Pediatr Endocrinol Metab 2016;29:769-75.

27. Pastor A, Conn J, Teng J, O’Brien CL, Loh M, Collins L, et al. Alcohol and recreational drug use in young adults with type 1 diabetes. Diabetes Res Clin Pract 2017;130:186-95.

28. MacNaught N, Holt P. Type 1 diabetes and alcohol consumption. Nurs Stand 2015;29:41-7.

29. Naimi TS, Stadtmueller LA, Chikritzhs T, Stockwell T, Zhao J, Britton A, et al. Alcohol, age, and mortality: estimating selection bias due to premature death. J Stud Alcohol Drugs 2019; 80:63-8.

30. Lunstead J, Weitzman ER, Harstad E, Dedeoglu F, Gaffin JM, Garvey KC, et al. Screening and counseling for alcohol use in adolescents with chronic medical conditions in the ambulatory setting. J Adolesc Health 2019;64:804-6.

31. Barnard K, Sinclair JM, Lawton J, Young AJ, Holt RI. Alcoholassociated risks for young adults with Type 1 diabetes: a narrative review. Diabet Med 2012;29:434-40.

32. Pinquart M, Shen Y. Anxiety in children and adolescents with chronic physical illnesses: a meta-analysis. Acta Paediatr 1992; 100:1069.

33. Pinquart M, Shen Y. Depressive symptoms in children and adolescents with chronic physical illness: an updated metaanalysis. J Pediatr Psychol 2011;36:375-84.

34. Farabi SS. Type 1 diabetes and sleep. Diabetes Spectr 2016;29: 10-3.

35. van Dijk M, Donga E, van Dijk JG, Lammers GJ, van Kralingen KW, Dekkers OM, et al. Disturbed subjective sleep characteristics in adult patients with long-standing type 1 diabetes mellitus. Diabetologia 2011;54:1967-76.

36. Caruso NC, Radovanovic B, Kennedy JD, Couper J, Kohler M, Kavanagh PS, et al. Sleep, executive functioning and behaviour in children and adolescents with type 1 diabetes. Sleep Med 2014; 15:1490-9.

37. Hirshkowitz M, Whiton K, Albert SM, Alessi C, Bruni O, DonCarlos L, et al. National Sleep Foundation's sleep time duration recommendations: methodology and results summary. Sleep Health 2015;1:40-3.

38. von Schnurbein J, Boettcher C, Brandt S, Karges B, Dunstheimer D, Galler A, et al. Sleep and glycemic control in adolescents with type 1 diabetes. Pediatr Diabetes 2018;19:143-9.

39. Jaser SS, Foster NC, Nelson BA, Kittelsrud JM, DiMeglio LA, Quinn M, et al. Sleep in children with type 1 diabetes and their parents in the T1D exchange. Sleep Med 2017;39: 108-15.

40. Sinisterra M, Hamburger S, Tully C, Hamburger E, Jaser S, Streisand R. Young children with type 1 diabetes: sleep, healthrelated quality of life, and continuous glucose monitor use. Diabetes Technol Therapeut 2020;22:639-42. 